Simulation of optical near and far fields of dielectric apertureless scanning probes

This article has been downloaded from IOPscience. Please scroll down to see the full text article.

2006 Nanotechnology 17475

(http://iopscience.iop.org/0957-4484/17/2/022)

View the table of contents for this issue, or go to the journal homepage for more

Download details:

IP Address: 134.105.184.12

The article was downloaded on 24/01/2012 at 08:09

Please note that terms and conditions apply. 


\title{
Simulation of optical near and far fields of dielectric apertureless scanning probes
}

\author{
R Esteban, R Vogelgesang and K Kern \\ Max Planck Institute für Festkörperforschung, Stuttgart, Germany \\ Received 18 May 2005, in final form 15 November 2005 \\ Published 21 December 2005 \\ Online at stacks.iop.org/Nano/17/475
}

\begin{abstract}
We study apertureless field enhancing optical probes beyond the spherical approximation in a smooth transition towards up to $3 \mu \mathrm{m}$ long conical silicon tips. Such tips are used in apertureless scanning near field optical microscopy, which holds the promise of sub $10 \mathrm{~nm}$ lateral resolution. A fully three-dimensional numerical solution to the Maxwell equations is obtained with the multiple multipole method giving simultaneously both near fields and scattered far fields. The significance of focused beam excitation for work with long tips is illustrated and the relative influence of relevant length scales such as tip length, excitation wavelength, and beam waist radius is discussed. In the limit of vanishing tip apex radius, the near field grows without bounds, whereas the far field remains finite. We verify that for small apex radii the near field confinement at the tip apex, which is related to the achievable lateral resolution, scales with the inverse of the radius. We find, however, that long tips exhibit a markedly lower confinement than spherical or very short tips. Relevant for experimental studies, we demonstrate how scanning the excitation field with long conical tips can be a useful technique for mapping the focal volume. We show that the normalized near field at the tip apex is robustly tolerant against small misalignments or misorientations of illumination focus and tip apex.
\end{abstract}

\section{Introduction}

One of the most promising optical imaging techniques for achieving lateral resolution of a few nanometres is the scattering type apertureless scanning near field optical microscope (aSNOM) [1, 2]. It utilizes localized, strong near field enhancement at the apex of a scanning probe tip, belonging typically to a scanning tunnelling microscope (STM) or an atomic force microscope (AFM). The local optical response of a sample can be determined with a lateral resolution essentially given by the effective apex radius. Images based on elastic scattering have been demonstrated at frequencies ranging from the visible to the microwave [3-5]. Inelastic spectroscopic phenomena such as Raman scattering and photoluminescence are also accessible [6, 7].

There are two limiting modes of operation of an aSNOM, namely, as a passive re-scatterer of local optical fields into the far field [8] and as an active source whose own localized near field excites an optical response of exclusively those sample volumes that are in close proximity to the tip apex [6]. In either case, as the probe tip scans a sample surface, changes in the far field can be observed which are related with the optical properties of the sample volume just below the tip, as well as local topography, and instrumental parameters [9-11].

In general, neither the passive nor the active aSNOM mode is realized in its pure form, because the optical responses of the probe tip and sample surface also interact with each other and require one to consider the tip-sample system as a whole. If the localized field enhancement at the tip apex acting as an active source is strong enough to overwhelm the parasitic scattering from the surrounding area and the bulk of the tip that enters the far field collection optics, a direct recording of the local optical properties is possible. Otherwise, a sophisticated amplification and discrimination scheme has to be employed [12-15].

A pivotal assumption for field enhancing near field optical microscopy is that the observable far field is directly related (proportional in the simplest case) with the fields at the apex of the field enhancing tip. In other words, the bulk of the tip may effectively be disregarded. Correspondingly, this nearto-far field transition is frequently taken to be appropriately modelled by representing the whole tip as a dipole polarizable sphere centred in the spherical apex cap featuring a scalar or 
tensorial polarizability $[16,17]$. Spectroscopic trends which enter through the wavelength dependence of the dielectric constant were found to be adequately captured by these simple models. Simulating a spherical tip can give important additional insights [18] but it was soon found that the actual tip shape may indeed have considerable impact on the near field enhancement and observable far field intensities [19-23].

In experimental aSNOM work one frequently uses tips which are metal-coated or even fully metallic. Their negative real part of the dielectric constant can lead to polariton resonances which increase the attainable signal considerably. Especially for nanoscopic structures, however, these are very sensitively coupled with the exact geometry and relative orientation of tip and sample through the phenomenon of quasistatic resonances [23]. In this paper, we wish to concentrate on those geometrical aspects that are decoupled from such resonances. Therefore, we choose a positive real part dielectric material like silicon at visible frequencies. Our second motivation for simulating $\mathrm{Si}$ tips concerns the achievable spatial resolution of aSNOM instruments, which is related to the tip apex radius. In this context, Si tips are a prime candidate for ultimate resolution well below $10 \mathrm{~nm}$ thanks to processing technology readily available for semiconductors but not for metals. Preliminary experimental results demonstrated to us the feasibility of using Si tips.

For the principal geometry, which is closely related to our own experimental set-up, refer to figure 1. First, conical tips of a wide range of apex radius and length are studiedup to $3 \mu \mathrm{m}$ in length-which establishes relevant model parameters for realistic elongated tips to facilitate subsequent studies of full tip-sample models (to be published separately). Afterwards, for long enough tips, the parameters defining the incident radiation beam are studied, namely, the angle of incidence, the Gaussian beam waist radius and the position of the tip relative to the optical focus. The results obtained are significant for optimizing experimental set-ups and exemplify how certain model simplifications, when overdone, may lead to discrepancies with respect to experimental results.

We stress the significance even of isolated tips as representative models for the passive mode aSNOM. In conjunction with the spatially variable field of a focused beam excitation we determine the relation of local fields and observable far field quantities. This allows us to verify the fundamental assumption for passive mode aSNOM that the scattered far field intensity scales with the local excitation field at the tip's apex [8]. It also suggests a means to experimentally characterize the incident beam and align the tip in the proper focal position.

\section{Simulated model}

In our model, we study the interaction of a focused Gaussian illumination beam with conical tips of up to $3 \mu \mathrm{m}$ length, whose hemispherical apex has a radius as small as $2 \mathrm{~nm}$. Thus it belongs to a class of multi-scale electrodynamic models that presents a number of challenges [24]. An elegant and numerically not too demanding solution is obtained with the generalized multipole technique (GMT) [25-27].

The GMT divides space into domains of different material properties, with the interfaces separating the domains as (a)

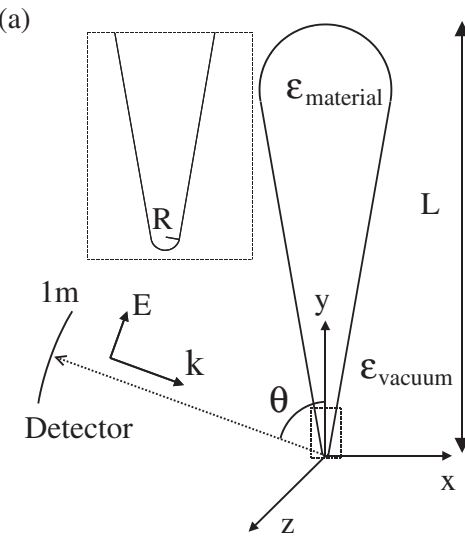

(b)

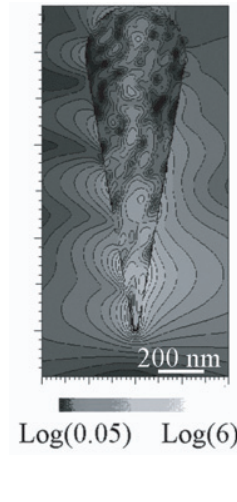

Figure 1. Geometry of the model tips considered and example of field patterns obtained. (a) Conical Si tips of length $L$ and apex radius $R$ are illuminated in vacuum by a Gaussian beam at an angle $\theta$ with respect to the tip axis. The scattered far field is recorded in backscattering direction. (b) Typical simulation result exhibiting the near fields outside a long tip and a standing wave pattern inside with a characteristic length scale $\sim \lambda_{\text {matter }}$. A logarithmic scale is used because linear contrast would allow us only to observe the strongest fields near the apex. The isolines represent an increase of $\times 1.3 \mathrm{in}$ the field modulus.

boundaries. It uses a set of expansion series of the electromagnetic field, for example multipoles or planar waves, which are perfect solutions to the three-dimensional Maxwell equations. The field in the domain is represented by one or more such expansion series, which are parameterized by an appropriate number of coefficients. These coefficients are the free parameters of the model that need to be determined, for example, by least-squares minimization of the error in the boundary conditions at the discretized domain interfaces. These technical aspects of the modelling are largely automated in the numerical platform MAX-1 that we use [28]. Still, the location and type of the different expansion series must be selected with care to avoid interdependences.

The geometry of the simulated tip and illumination as well as collection optics is shown in figure 1(a). Unless stated differently in the text, the following parameters apply. The tips are of conical shape, with a half angle of $10^{\circ}$. The upper and the lower ends of the cone are capped by two hemispheres, forming a convex $C^{1}$ continuous surface. The radius of the smaller cap (the tip apex) is $10 \mathrm{~nm}$. The tip material is silicon $\left(\epsilon_{r}=17.76+0.508 \mathrm{i}[29]\right)$, embedded in vacuum.

The illumination is a Gaussian beam of $\lambda_{\mathrm{vac}}=514 \mathrm{~nm}$, corresponding to one of the principal wavelengths of an $\mathrm{Ar}^{+}$ ion laser. The implementation in the program corresponds to a fifth-order correction of the paraxial Gaussian beam [30-32] and is accurate near the focus for not too tight beams. The beam is in-plane linearly polarized with an angle of incidence $\theta$ with respect to the axis of the tip of $70^{\circ}$ and a waist radius $500 \mathrm{~nm}$. To illustrate the simulations, figure 1(b) presents the distribution of the electric field for a $1.4 \mu \mathrm{m}$ long tip.

In the different results presented, the excitation radiation is suppressed and only the values of the scattered near and far fields are considered, as they are the ones of interest in aSNOM. The near field enhancement is defined as the ratio of the maximum average field modulus of the excited field 
Simulation of optical near and far fields of dielectric apertureless scanning probes

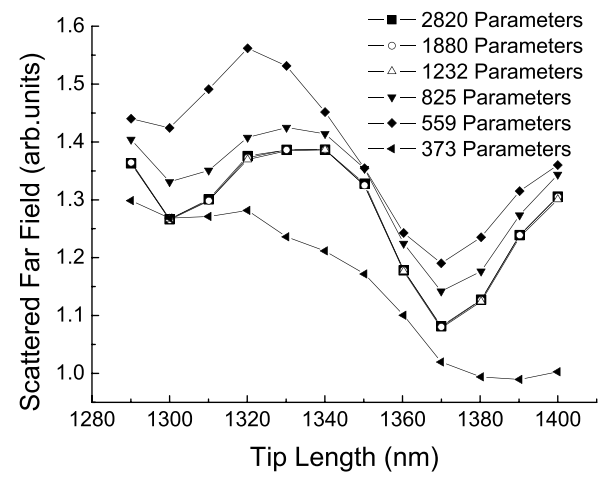

Figure 2. Study of the convergence of the scattered far field when the number of parameters is increased, at tips of different lengths.

(evaluated near the apex) to the maximum field modulus of the incident beam. The back-scattered far field is obtained by integrating the average Poynting vector in a circular detector covering a solid angle of $\mathrm{NA}=0.17$, centred in the trajectory of the backscattered beam (figure 1). The simulated distance to the tip is one metre, to guarantee that only the far field is measured.

We apply two simultaneous criteria for acceptable convergence of the results. First, we accept only results with an average relative error along the boundary less than $0.5 \%$. The error must not be significantly affected when other boundary points are evaluated than those used in the error minimization. Second, in each data set assembled to one graph we checked for a significant number of models the effect of increasing the total number of free parameters in the field expansions by $\sim 50 \%$. The variation in the obtained near field enhancement and scattered far field intensity must be less than $1 \%$. We took special care in the limiting cases of the model considered. Figure 2 serves to illustrate the good convergence of the results, by showing the evolution of the scattered far field with the number of parameters for typical tips of lengths between 1.3 and $1.4 \mu \mathrm{m}$. In this particular example, our two criteria are first verified by the curve with 1232 free parameters.

\section{Results}

\subsection{Influence of tip shape}

In figure 3(a), the influence of the length $L$ of the tip on the near field enhancement and scattered far field is shown for the case of Gaussian illumination. Three regions of qualitatively different behaviour can be identified, namely a monotonic increase for lengths less than $\lambda_{\text {vac }} / 2$, an oscillatory behaviour between $\lambda_{\text {vac }} / 2$ and $2 \mu \mathrm{m}$, and an approximately constant behaviour beyond that.

Starting from a length of $20 \mathrm{~nm}$, i.e., a sphere of $10 \mathrm{~nm}$ radius, the near field enhancement increases almost linearly with the length $L$ due to a mostly constructive contribution originating in all the material, up to $L \approx 200 \mathrm{~nm}$. We found that in this tip length range the scattered far field intensity scales approximately with the volume $V$ in a power law with exponent 2.55 , which contrasts with the usual expectation derived from Rayleigh scattering theory, predicting a $V^{2}$ behaviour for small particles [33]. This we traced to the shape being changed in the transition from an elongated conical shape $(L=200 \mathrm{~nm})$ to a spherical tip $(L=20 \mathrm{~nm})$. If instead the tip is scaled uniformly, preserving the conical shape, we obtain the familiar $V^{2}$ law.

Beyond $L \approx 200 \mathrm{~nm}$ resonances appear. They are related to the length scales given by the wavelengths outside and inside the tip on the one hand and tip size parameters on the other-notably the tip length and, as long as it is strongly illuminated, the radius of the top hemispherical cap. The first maximum corresponds to a length slightly bigger than $\lambda_{\text {vac }} / 2$, i.e., the first resonance value expected for linear antennas [34]. Such behaviour has already been observed in the equivalent two-dimensional case [35]. As the tip gets bigger, the resonances become less pronounced, though they are still non-negligible even at lengths of around $1 \mu \mathrm{m}$. In this region, the resonance length $\lambda_{\text {matter }} / 2$ appears more important, in correspondence with the standing wave patterns that form as illustrated in figure 1(b). The Fourier transform of the near field enhancement and scattered far fields for tips between 1 and $1.8 \mu \mathrm{m}$ (not shown here) indicates a high spectral density close to $\lambda_{\text {matter }} / 2$. This is especially clear in the simulation of artificial tips of real and positive $\epsilon_{r}$, which show no damping of internal waves.

At tip lengths of around six times the illumination beam waist radius, the hemispherical top cap is not efficiently illuminated anymore and the waves generated near the tip are damped away before they are reflected at the top. The results converge to an almost constant level. Between 2.8 and $3 \mu \mathrm{m}$, the variation is less than $\pm 4 \%$.

In figure 3(b) the same results are shown as in (a) except for the use of planar wave excitation. In this case the whole tip is always fully illuminated, including the hemispherical top cap. For small lengths, the results are almost identical to those obtained with focused Gaussian illumination. However, for bigger lengths the use of planar illumination affects the results dramatically, making it very difficult to extrapolate to an 'infinite tip'. The change is especially accentuated for the scattered far field, as the influence of the hemispherical top cap dominates the results.

The second tip shape parameter we studied is the radius of its apex. Realistic probe tips feature effective radii of significantly less than $100 \mathrm{~nm}$, good ones less than $10 \mathrm{~nm}$. Figure 4 shows the evolution of the near field enhancement and the scattered far field intensity with the apex radius in this range of interest. The length of the tip is kept constant at $3 \mu \mathrm{m}$, where the influence of length-related resonances is nearly negligible. For radii below $\sim 30 \mathrm{~nm}$ the symmetric point exactly below the tip apex is also the location of the strongest near field. Above $\sim 30 \mathrm{~nm}$, however, two values of enhancement-the actual maximum and the value just below the tip — are shown because the location of the field maximum is shifted significantly away from the exact apex of the tip due to retardation effects. As expected, the near field enhancement diverges towards zero radius. The expression $13.2-2.83 \ln (R / \mathrm{nm})$ describes the near field enhancement quite accurately for radii $R$ between 2 and $30 \mathrm{~nm}$. The scattered far field also increases for smaller radii, although it does not diverge but it saturates.

Not only are the scattered intensity and the maximum of the near field modulus of interest but also the local distribution and field lines of the near field. Figure 5(a) presents the 

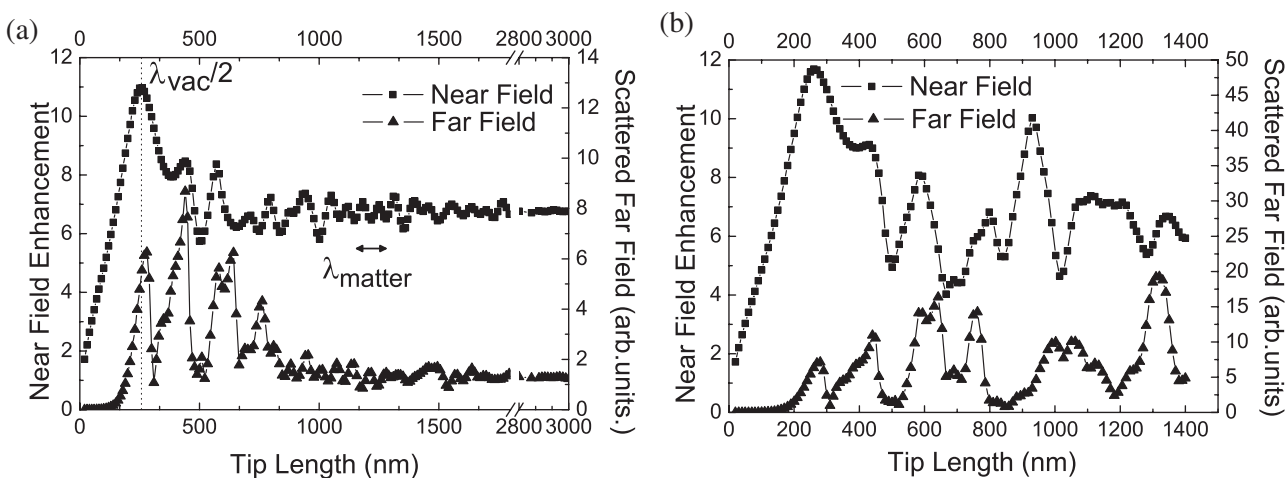

Figure 3. Near field enhancement and scattered far field when the length of the tip is modified, for Gaussian beam (a), and planar wave (b) illumination.

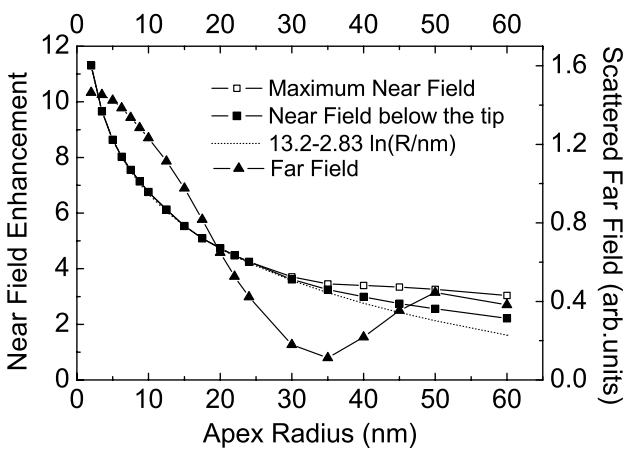

Figure 4. Evolution of the near and far field for apex radius varying between 2 and $60 \mathrm{~nm}$.

strength of the field $E$ along horizontal lines below the apex of the tip. In the vicinity of the apex, it is predominantly vertically oriented-along the $y$ axis. Notice the reduction of the confinement when the vertical distance from the tip is increased (figure 5(b)). At a $5 \mathrm{~nm}$ distance, a full width at half maximum of $\sim 32.5 \mathrm{~nm}$ is obtained, to be compared with $\sim 18.5 \mathrm{~nm}$ just below the apex. As the confinement of the field is related to the resolution achievable when scanning a sample, it is favorable to use a very small tip-substrate distance. For tips longer than a few hundred nanometres the shape of the apex fields is essentially independent of length. Very small tips, however, produce more confined fields than longer tips, both in the horizontal and the vertical direction [27]. For example, the full width at half maximum just below the tip is $\sim 13.5 \mathrm{~nm}$ for the sphere. Evidently, simulating small or even spherical tips may overestimate the resolution by several tens of per cent.

The normalized fields scale spatially quite accurately with the radius, as shown in figure 5, where the shapes of the field in a horizontal line at $R / 2$ below the tip are plotted, with $R$ being the radius of the tip. The vertical distance is chosen as $R / 2$ to illustrate how the results scale with $R$ not only horizontally but also vertically, for distances of the order of $R$.

We note that the results converge surprisingly well to a Lorentzian shape for tips of a few hundred nanometres length or more. They are proportional to

$$
|E(x)| \propto E_{0}+\frac{1}{Y_{0}^{2}+x^{2}}
$$

where $E_{0}$ and $Y_{0}$ are fitting parameters. $Y_{0}$ is of the order of $R$ and we have found it to depend only weakly on the illumination properties (the beam radius and others). Moreover, we observed an excellent rotational symmetry of the solution in the horizontal $x z$ plane below the apex which suggests further generalization to a cylindrically invariant expression [36].

\subsection{Influence of the illumination}

In this subsection, the influence of the illumination used is studied. When considering not just a planar wave excitation, but Gaussian illumination, besides the angle of incidence and polarization, the Gaussian beam waist radius and the relative location of tip apex and focus centre are relevant parameters. Qualitatively, the consequences can be understood as collective retardation effects, with different parts of the whole tip volume oscillating in phase. Depending on the details of how a specific illumination beam excites the different partial volumes, their overall interference may be constructive or destructive. Sensitive dependence of this interference on beam waist radius and angle of incidence $\theta$ of the beam can be expected.

Figure 6 shows the effect of variable beam waist radius, keeping constant the amplitude at the focus. For very tight illumination, under the standard conditions defined above $\left(\theta=70^{\circ}\right)$, the near field enhancement and scattered far field are maximal. Destructive overall interference from excitation of a larger tip volume reduces the fields for growing radii [37]. As can be seen from figure 6, whereas the near field at the apex continues to fall for increasing radii, the far field intensity exhibits a local minimum around $700 \mathrm{~nm}$ radius and is dominated eventually by the hemispherical region at the top of the tip. As expected, in the limit of infinite beam waist radius both the near and far field values converge to the planar wave illumination case.

For $\theta=20^{\circ}$ (not shown here), we find the trend quite altered in the sense of a more constructive excitation of larger tip volumes. From a minimum in near and far field scattering for tight illumination both intensities increase monotonically towards their respective plane-wave excitation values.

Figure 7 shows the influence of changing $\theta$ on the field strengths for a $3 \mu \mathrm{m}$ long tip. The near field enhancement is minimal for $\theta=0^{\circ}$ and $180^{\circ}$ when the linear polarization vector of the electrical field is perpendicular to the axis of 
Simulation of optical near and far fields of dielectric apertureless scanning probes
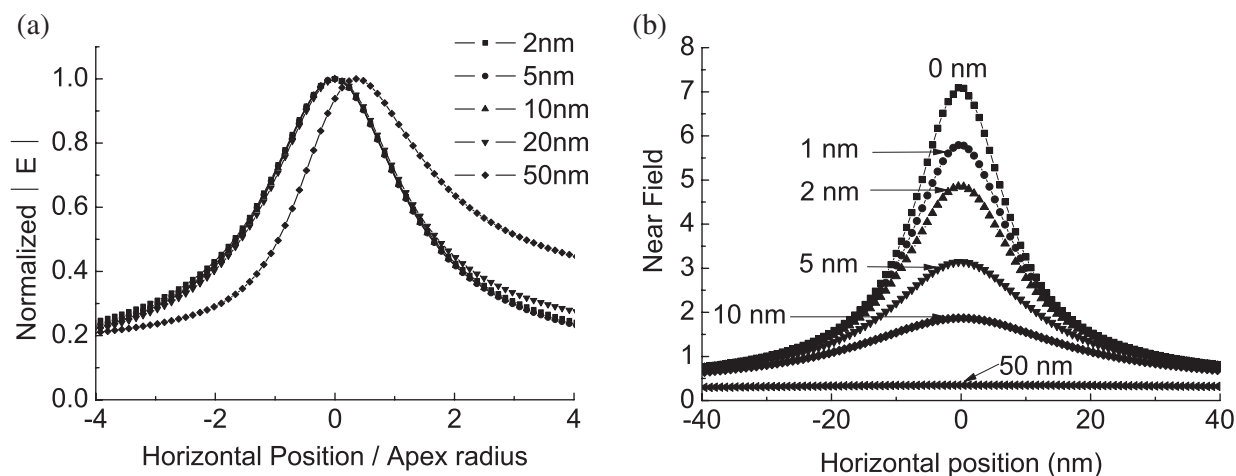

Figure 5. (a) Near field modulus (normalized to the maximum) along a horizontal cut at $R / 2$ below the apex for different radii. (b) Near field modulus (relative to the field modulus at the focus of the incident radiation) for $R=10 \mathrm{~nm}$ along horizontal lines located at different distances below the tip apex.

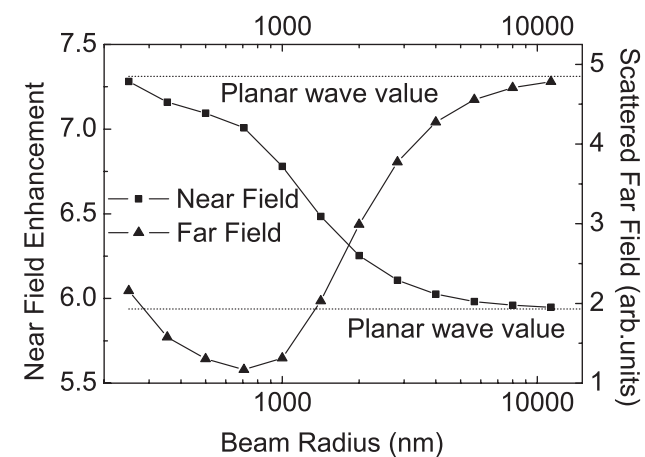

Figure 6. Near field enhancement and scattered far field intensity for different radii of the Gaussian excitation beam, asymptotically approaching those of a planar wave excitation for increasing beam waist radius.

the tip [36, 38]. The maximum is however not observed at $90^{\circ}$ (where the electrical field vector is exactly parallel to the axis of the tip) but at a different angle [39], here $\theta \sim 40^{\circ}$. The scattered far field intensity presents four maxima. One corresponds to the near field enhancement maximum at $\sim 40^{\circ}$ and two to the direct illumination of the top hemispherical cap - already seen to be strongly radiating, but of little relevance to experimental measurements. The fourth maximum, located at $\sim 100^{\circ}$, corresponds to illumination perpendicular to the side surface of the cone. The behaviour of the normalized modulus of the electric fields near the apex is approximately constant for $\theta=30^{\circ} \ldots 180^{\circ}$.

The near field enhancement and far field intensity when the beam focus is not centred at the apex but displaced in the $y$ direction is presented in figure 8. As long as the top cap of the tip is much less illuminated than the apex of the tip (here $y \lesssim$ waist radius), both values scale quite closely with the local field of the excitation at the apex of the tip-the square of the field modulus in the case of the scattered far field intensity. This suggests an appealing way of characterizing the beam used in experimental set-ups [17]. Equivalent conclusions are obtained when displacing the illumination beam in the $z$ and $x$ directions. The normalized near field modulus near the apex remains approximately unchanged, as long as the tip apex remains in the focal volume given by the waist radius and the

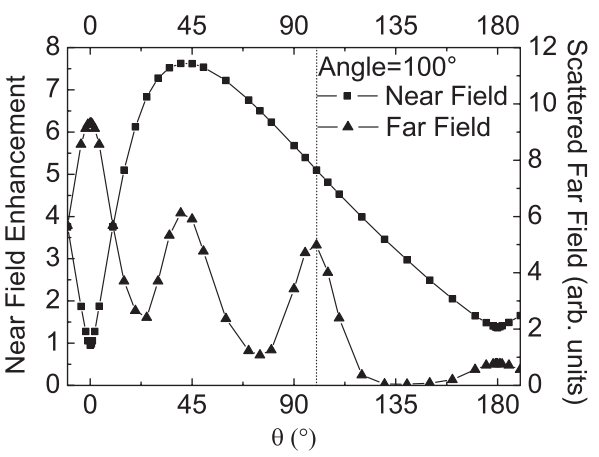

Figure 7. Evolution of the results as a function of the angle $\theta$ between the beam propagation direction and the axis of a $3 \mu \mathrm{m}$ tip. Near field enhancement and scattered far field intensity. A local maximum around $\theta \sim 40^{\circ}$ is found.

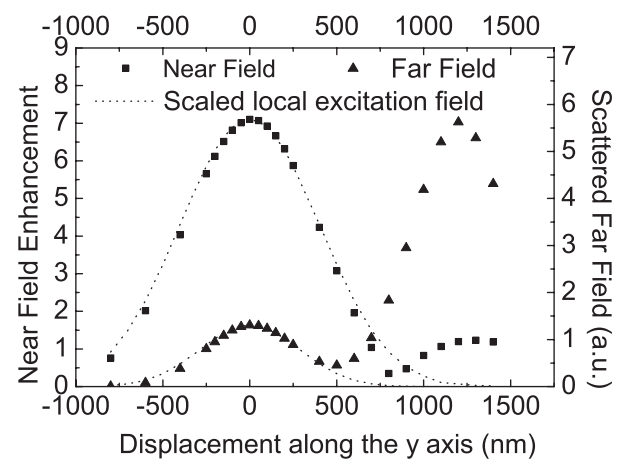

Figure 8. Near field enhancement and scattered far field intensity for displacements of the focus along the $y$ axis. Zero displacement corresponds to the location of the apex of the tip. Displacements towards positive values correspond to illumination of the upper part of the tip. The secondary maxima at $\sim 1.25 \mu \mathrm{m}$ are related to the direct illumination of the top cap. For comparison, we plot with a dotted line the appropriately scaled modulus of the excitation field at the apex of the tip with the near field enhancement and its square with the scattered far field intensity for the corresponding displacement of the focus.

Rayleigh length. Thus, small misalignments will likely not critically affect the images obtained when scanning a sample. 


\section{Discussion}

The near field enhancement obtained at the apex of sufficiently long Si tips upon illumination with focused $514 \mathrm{~nm}$ radiation settles at a value around 7. This is in the low range of field enhancements reported in related studies, which go from values less than 10 up to more than 70 [35, 36, 40, 41]. The difference derives partly from the different materials and shapes of the tips, but mostly from the presence or absence of geometrical and plasmon or other polariton resonances. The present study concentrates on the effects of tip geometry and the consequences of variations in the alignment of the exciting radiation. For $\mathrm{Si}$ at visible frequencies the real part of the dielectric constant is large and positive, exhibiting no polariton resonances that would affect the results.

Different length scales are relevant to this study. Only for sufficiently long tips and only for focused illumination do we find near field enhancement and far field scattering intensity to reach values that are stable against further increases of the tip length. That is, the ratio of beam focus and tip length plays an important role. A planar wave excitation can be inappropriate in a simulation when the scattered far field intensity is desired, as the results are likely dominated by the bulk or the upper termination of the tip and give little information about the fields at the apex of the tip. From additional studies we find that the dampening effect of the imaginary part of the index of refraction - that is, the ratio of penetration depth to tip lengthappears to be equally important. A small but finite extinction coefficient ensures that waves generated near the apex do not travel too far inside the domain and form extended standing wave patterns. Otherwise these would lead to an increased sensitivity of the near and far fields to changes in tip geometry and illumination parameters as is characteristic of undamped resonance phenomena.

The simulation of very short tips or the extreme case of spherical tips leads to somewhat misleading results as they significantly underestimate the far field intensity and may overestimate the achievable resolution. The greater field confinement of short tips can be analysed by considering the radial distance dependence of the near field intensity, which is critical in sample scanning, where the tip-substrate interaction depends sensitively on the distance $r$. The electric near field of a simple dipole polarizable sphere scales as $r^{-3}$. In contrast, equation (1) suggests an $r^{-2}$ behaviour relative to an appropriately chosen origin inside the conical tips with spherical apex studied here. Comparing our result to the near fields at the point tip of perfect infinite cones, which are known to exhibit an $r^{\nu-1}$ behaviour with the critical exponent $v>0$ depending on the cone angle as well as the dielectric material [42], we believe the Lorentzian behaviour in our case is coincidental. For other cone angles, we expect the exponent to differ from -2 , which will be the subject of further studies. Nevertheless, the different distance scaling behaviours of the near fields for spherical and long tips could particularly affect the dynamic variants of scattering-type near field optical methods, which periodically modulate the probesample distance.

The observed antenna-like resonance at a tip length of $\sim \lambda_{\text {vac }} / 2$ verifies the possibility of specifically designed superresonant field enhancement structures [20]. Their realization, however, requires a precise manufacturing process and the excess near field enhancement of only some $60 \%$ over the long-tip value makes the effort questionable for this particular structure. Furthermore, the increase in the observable far field scattered intensity (more than four times the value of very long tips) results from all of the small tip's volume, and the contribution from only the local near field at the apex might be even more difficult to extract experimentally than in the case of long tips.

Over a wide range of apex radii, the field confinement scales inversely proportionally to the radius. Reducing the radius will likely result in a proportional improvement in resolution. Moreover, the scattered far field increases with reducing radius, which suggests that highly sharpened tips are also desirable for optimizing the received signal. The far field increase, however, remains finite and does not diverge as the near field enhancement, for reasons of energy conservation, which allows infinite near fields in an infinitesimal volume, but requires finite cross sections for the scattered far fields (figure 4).

The consideration of Gaussian beams in addition to planar wave excitations opens the possibility of maximizing near field enhancement and scattered far field by varying the characteristics of the excitation. For example, the finding of an optimal angle of incidence (figure 7), which differs from the case of polarization exactly along the tip axis, is relevant to the implementation of near-field optical microscopes, especially for opaque samples, which have to be illuminated under similar conditions. However, it is necessary to keep in mind that the presence of a substrate will in general modulate the optimal angle due to the exciting radiation resulting from interference of the incident beam with the waves reflected by the substrate surface.

Including the substrate is a substantial step for further studies. Nonetheless, we expect many of our present results to carry over to such simulations. Examples are: the need of simulating confined illumination for long tips, significant antenna resonances for tip lengths less than at least a few $\mu \mathrm{m}$, the considerable increase of near field enhancement and field confinement for decreasing apex radius, and the presence of a maximum for illumination polarization not parallel to the tip axis.

An important aspect of the present paper is the study of off-apex illumination, because it serves as a test of how near field properties can be related to those recorded in the far field in the passive aSNOM mode. Figure 8 illustrates how, under certain circumstances, a faithful near-to-far field transition is indeed facilitated by the use of near field enhancing tips. The experimentally accessible scattered far field intensity scales quite accurately with the square of the local field modulus of the excitation fields at the apex. This holds in our tips as long as the top cap is not strongly excited in comparison with the volume near the apex. With respect to the essential nearto-far field scattering relation being proportional, the simple spherical models are thus validated.

Two caveats must be noted, however. First, the gradient of the local excitation fields used in the present study is small on the length scale of the tip apex radius, i.e., $|\partial / \partial \mathbf{x}| \ll$ $1 / R$. At this point it is still an open question how well the intensity a far field detector registers corresponds to the 
Simulation of optical near and far fields of dielectric apertureless scanning probes

local fields at a sharp tip probing near fields that vary with equally high spatial frequency. Second, as already mentioned, when the tip approaches a substrate it exhibits both active and passive behaviour. Considering the tip-substrate interaction considerably complicates the study. In this scenario, the relation between the local excitation field and the scattered far field intensity is likely no longer a simple proportionality.

Finally, we emphasize the immediate applications of our results to the experimental work. As long as the upper part of the tip is not overilluminated, the simple relationship between excitation fields, local near fields, and scattered far fields allows one to map the three-dimensional focus by scanning the tip apex through the focal volume. That is, this method constitutes a convenient method for three-dimensional beam focus characterization. Also, the maximum found when the excitation is focused at the apex greatly simplifies the alignment of experimental set-ups like that of figure 1, an approach successfully employed in the alignment of our own experimental set-up. Further, it can be observed that slight off-focus alignment of less than the focal waist radius or small variations of the angle of incidence of a Gaussian beam exciting the apex of long tips do not affect the near fields at the apex too much. Apart from a uniform scaling factor, the spatial field structure is rather tolerant against somewhat sub-optimal alignment of the excitation.

\section{Conclusion}

In this paper we studied how both the scattered near and far fields of optically excited conical tips evolve with tip length and apex radius, with the angle of incidence and the waist radius of the Gaussian excitation beam, as well as with the relative alignment of tip apex and beam focus.

The near field enhancement and scattered far field intensity vary considerably when tips are considered that are short compared to the beam waist radius and the penetration depth but level out for long enough tips. Accordingly, appropriately long tips should be used together with sufficiently focused excitation beams to obtain steady behaviour for both the near field enhancement at the tip apex and the recorded far fields. The simulation of too short or spherical tips also results in more confined fields, which can lead to overestimating the achievable lateral resolution as well as a misjudgment of the distance dependence of the near field mediated tip-sample interaction.

Reducing the apex radius gives rise to a proportional increase in near field confinement, which is expected to translate to better lateral resolution. Our simultaneous modelling of near and far field scattering demonstrates how for vanishing radius the near field enhancement grows without bounds. But the detectable scattered far field intensity, that is, the experimentally relevant quantity, remains finite in accordance with energy conservation.

We found an optimal angle of incidence, $\theta \sim 40^{\circ}$, for maximal near field strength as well as scattered far field intensity. Note that this does not realize an electric field vector parallel to the isolated tip's axis. Different tip materials or geometries, of the presence of a sample surface, whose reflections also excite the tip, will likely alter the optimal angle, requiring further numerical studies.
Finally, the direct correspondence between scattered far field intensity and the square modulus of the incident field at the apex suggests a convenient method for three-dimensional focus characterization as well as alignment of tip apex and optical focus. The near field spatial distribution around the tip apex is also rather tolerant against small misalignments or misorientations, with only a uniform reduction in field strength. This suggests that field enhancing near field optical microscopy may indeed be developed into a robust tool for routine investigations of optical properties at the nanometre scale.

\section{References}

[1] Zenhausern F, Martin Y and Wickramasinghe H K 1995 Science 2691083

[2] Knoll B and Keilmann F 1999 Nature 399134

[3] Knoll B, Keilmann F, Kramer A and Guckenberger R 1997 Appl. Phys. Lett. 702667

[4] Keilmann F, Knoll B and Kramer A 1999 Phys. Status Solidi b 215849

[5] Taubner T, Hillenbrand R and Keilmann F 2003 J. Microsc. 210311

[6] Hartschuh A, Sanchez E J, Xie X S and Novotny L 2003 Phys. Rev. Lett. 90095503

[7] Hu D, Micic M, Klymyshyn N, Suh Y D and Lu H P 2003 Rev. Sci. Instrum. $\mathbf{7 4} 3347$

[8] Hillenbrand R, Keilmann F, Hanarp P, Sutherland D S and Aizpurua J 2003 Appl. Phys. Lett. 83368

[9] Madrazo A, Nieto-Vesperinas M and García N 1996 Phys. Rev B 533654

[10] Carminati R and Greffet J-J 1995 J. Opt. Soc. Am. A 122716

[11] Martin O J F, Girard C and Dereux A 1996 J. Opt. Soc. Am. A 131801

[12] Hillenbrand R and Keilmann F 2000 Phys. Rev. Lett. 853029

[13] Maghelli N, Labardi M, Patanè S, Irrera F and Allegrini M 2001 J. Microsc. 20284

[14] Walford J N, Porto J A, Carminati R, Greffet J-J, Adam P M, Hudlet S, Bijeon J-L, Stashkevich A and Royer P 2001 J. Appl. Phys. 895159

[15] Fikri R, Barchiesi D, H'Dhili F, Bachelot R, Vial A and Royer P 2003 Opt. Commun. 22113

[16] Koglin J, Fischer U C and Fuchs H 1997 Phys. Rev. B 557977

[17] Bouhelier A, Beversluis M R and Novotny L 2003 Appl. Phys. Lett. 824596

[18] Porto J A, Johansson P, Apell S P and López-Ríos T 2003 Phys. Rev. B 67085409

[19] Kottmann J P, Martin O J F, Smith D R and Schultz S 2001 Phys. Rev. B 64235402

[20] Krug J T II, Sánchez E J and Xie X S 2002 J. Chem. Phys. 11610895

[21] Calander N and Willander M 2002 J. Appl. Phys. 924878

[22] Renger S, Grafström J and Eng L 2005 Phys. Rev. B 71075410

[23] Fredkin D R and Mayergoyz I D 2003 Phys. Rev. Lett. 91253902

[24] Girard C and Dereux A 1996 Rep. Prog. Phys. 59657

[25] Hafner C 1999 Post-Modern Electromagnetics: Using Intelligent Maxwell Solvers (Chichester: Wiley)

[26] Moreno E, Erni D, Hafner C and Vahldieck R 2002 J. Opt. Soc. Am. A 19101

[27] Bohn J L, Nesbitt D J and Gallagher A 2001 J. Opt. Soc. Am. A 182998

[28] Hafner C 1998 Max-1: A Visual Electromagnetics Platform (Chichester: Wiley)

[29] Aspnes D E and Studna A A 1983 Phys. Rev. B 27985

[30] Davis L W 1979 Phys. Rev. A 191177 
[31] Barton J P and Alexander D R 1989 J. Appl. Phys. 662800

[32] Evers T, Dahl H and Wriedt T 1996 Electron. Lett. 321356

[33] Van de Hulst H C 1981 Light Scattering by Small Particles (New York: Dover)

[34] Collin R E and Zucker F J 1969 Antenna Theory (New York: McGraw-Hill)

[35] Crozier K B, Sundaramurthy A, Kino G S and Quate C F 2003 J. Appl. Phys. 944632

[36] Novotny L, Bian R X and Xie X S 1997 Phys. Rev. Lett. 79645
[37] Martin Y C and Wickramasinghe H K 2002 J. Appl. Phys. 913363

[38] Martin O J F and Girard C 1997 Appl. Phys. Lett. 70705

[39] Sun W X and Shen Z X 2003 J. Opt. Soc. Am. A 202254

[40] Martin Y C, Hamann H F and Wickramasinghe H K 2001 J. Appl. Phys. 895774

[41] Micic M, Klymyshyn N, Suh Y D and Lu H P 2003 J. Phys. Chem. B 1071574

[42] Van Bladel J 1991 Singular Electromagnetic Fields and Sources (Oxford: Oxford University Press) 


\section{Corrigendum}

Simulation of optical near and far fields of dielectric apertureless scanning probes

R Esteban, R Vogelgesang and K Kern Nanotechnology 17 475-482

The value given in line four of the first paragraph of page three for the numerical aperture used to collect the scattered radiation should read 'NA=0.342' instead of ' $N A=0.17$ '. The paper is otherwise unaffected. 